\title{
The Metaverse Phenomenon in the Teaching of Digital Media Art Major
}

\author{
Yong $\mathrm{Li}^{1,2}$ Dan Xiong ${ }^{3, *}$ \\ ${ }^{1}$ Beijing Institute of Technology, Zhuhai, Guangdong, China \\ ${ }^{2}$ Bangkokthonburi University, Bangkok, Thailand \\ ${ }^{3}$ Neusoft Institute Guangdong, Foshan, Guangdong, China \\ *Corresponding author. Email: xiongdan@nuit.edu.cn
}

\begin{abstract}
The interdisciplinary characteristics of the digital media art major have led to the combination of technology and art in practical teaching, especially the impact of technological changes on teaching. This article takes the metauniverse imagination that is the focus of teaching as an entry point, explains the influence of technological development on design teaching represented by digital media art from the perspective of own professional construction, teaching practice experience and teaching design management, and summarizes the problems that need to be addressed in future instructional design.
\end{abstract}

Keywords: Metaverse, Digital media art, Instructional design, Future education.

\section{INTRODUCTION}

The major of digital media art is an interdisciplinary design major with great demand for technological advancement and hardware. In teaching practice, students majoring in digital media art need to master professional training in artistic performance, and must also master the technical characteristics that support the development of art. In particular, the uncertainty that may arise between new technology and technological equipment and artistic creativity will bring teachers and students either fear or anxiety, or unlimited reverie. The former is mainly manifested as a kind of confusion and uncontrollable uneasiness in the unknown territory; while the latter is more inclined to romanticism and whimsical ideas that are not bound by any conditions. Regarding this feature, the more popular concept of "metaverse" might be thought of. This metaverse phenomenon in the professional teaching process will be discussed in the following content.

There is no exact concept about the actual

*Fund: Industry-University Cooperation Collaborative Education Projects of Ministry of Education: Online construction of a curriculum system corresponding to vocational positions based on TOPCARES methodology (No. 202002114005); Construction of a curriculum and practice base for digital media art majors (Project No. 202102199035). conceptual definition or practical form of the metaverse. People explain or define from different angles or understandings. Because of different starting points and positions, they will produce completely different or contradictory results [1]. However, this does not affect people's judgments on the possible outcomes of future technological development, that is, such uncertain results often have greater value for controversy and discussion [2]. This kind of discussion will generate more ideas and turn ideas into results. As for teaching, there is actually no real conclusion to support the teaching to complete the accurate description of the metaverse. The authors of this article are more inclined to it as a concept, which is the need for business development and operation. But if it is put in the category of technological innovation changing the future, it may affect the future setting of teaching content, including digital media art teaching, because the teaching is to be futureoriented after all.

\section{INTRODUCTION OF METAVERSE}

Then several questions need to be studied: What is the metaverse? What will the metaverse bring in the future? What kind of metaverse is needed in teaching? What kind of method do people need to achieve it? ("Figure 1") 


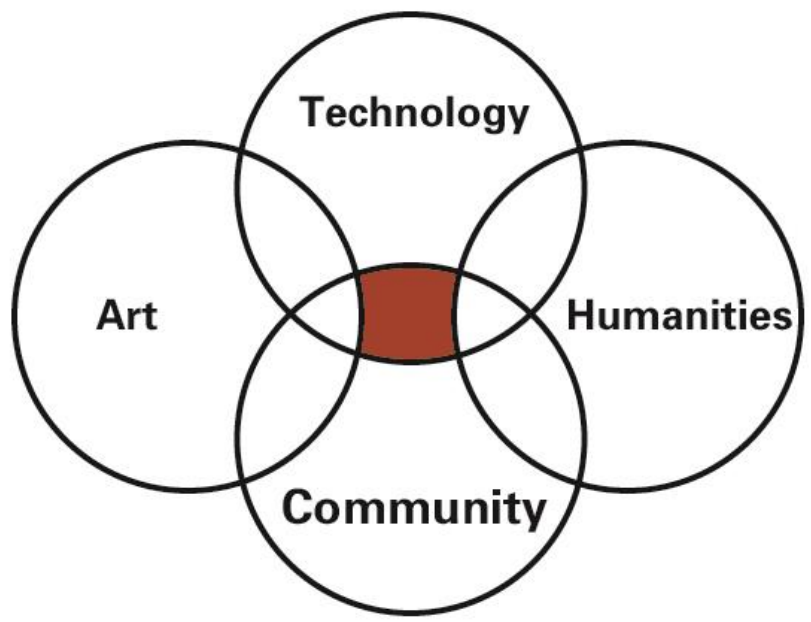

Figure 1 The metaverse.

How to define the metaverse in digital media? As mentioned earlier, in the face of new technological things, students will always have two diametrically opposed attitudes. Negative emotions are mainly reflected in the inability to accurately define it, or it is a kind of untouchable or perceptible, which will produce a sense of insecurity in learning. If it is necessary to solve the negative impact of this insecurity, the authors mainly emphasize the changes of technology to the future regarding the definition of "metaverse". But the next question students ask is that they need a specific method, skill, or channel. This phenomenon is also a conclusion that we easily come to in all teaching processes, that is, it can be explained at the theoretical level, but at the operational level, there is no way to achieve the output efficiency equivalent to the theory. Such a situation is contrary to the current rapid development of the media environment. Obviously, in the face of complex and changeable public opinion or at the level of information channels, the classroom cannot solve all the problems about innovation. Therefore, it is necessary to warn students that "metaverse" and all other innovative things represent the future, and the realization of the future depends on their imagination and the process of realizing the imagination.

So what will the metaverse bring in the future? If $5 \mathrm{G}$ network teaching can give full play to the advantages of network teaching during the epidemic, as a solution, 5G network teaching realizes the realism and immersion of long-distance teaching in buildings, and it is obvious that it is a further improvement in the existing teaching environment [3]. The question now is whether $5 \mathrm{G}$ network teaching is really that good. If there is a negative answer, it must be that distance teaching is difficult to achieve effective communication between people. This sense of distance will reduce the learning efficiency, and even have a serious impact on the health of young people, especially with regard to vision health. Therefore, it is obviously wrong to use VR or virtual technology to describe the existence of the metaverse whether it can truly replace the relationship between students and teachers in the classroom. Regarding the application of technology products, it is a must to first emphasize that it is still an auxiliary product, just like an autonomous driving system, it cannot really replace people to drive a traditional car. The significance of education itself is far greater than safe driving services. Therefore, from the level of teaching technical form or content realization, metaverse should assist the social relationship between students and teachers before people, instead of realizing virtual teaching scenes as the goal.

What kind of metaverse is needed in teaching? For example, it is mentioned that the teaching people need is a kind of social life with social temperature and emotion. If science and technology products lose humanistic care and only emphasize the sense of science and technology, it is obviously contrary to the meaning of human existence. So from this perspective, as a kind of high technology, it can not only produce future innovations, but at the same time it is full of contradictions and conflicts for human development. Therefore, what kind of metaverse is needed is a question worth 
discussing. In the real life perception, science and technology are people-oriented, and more attention is paid to the service of science and technology to people, rather than dominating or controlling. Then in teaching, the existence of the metaverse should also be used for teaching to provide services for teachers and students. Although it has an influence on people, we cannot lose our self-development under this influence. Just like bad kids who are addicted to online games, the pleasure brought by Internet technology has lost the joy of real life. Therefore, what people need is a metaverse that can inspire people's heart and self.

So what kind of method is needed to realize the metaverse? It is impossible for an applied profession, because in the future, what kind of situation that the specific form of the metaverse will become is still unknown to people. It is more like a forward-looking technological phenomenon than a specific product[4]. So in a sense, what people need is a yearning for the future. As for what it really will become, it is not that important.

\section{METAVERSE IN INSTRUCTIONAL DESIGN}

How to make this kind of scientific and technological phenomenon represented by the metaverse have stronger innovative applications in teaching? This article will take the four-year undergraduate professional courses set up by universities in mainland China as a case to explain. Here, the metaverse can be understood as a technological phenomenon or requirement. Students are required to have a clearer understanding of technology, using technology, and using technology to provide innovative services, and achieve self-improvement and the cyclical development of life through technology. ("Figure $2 ")$

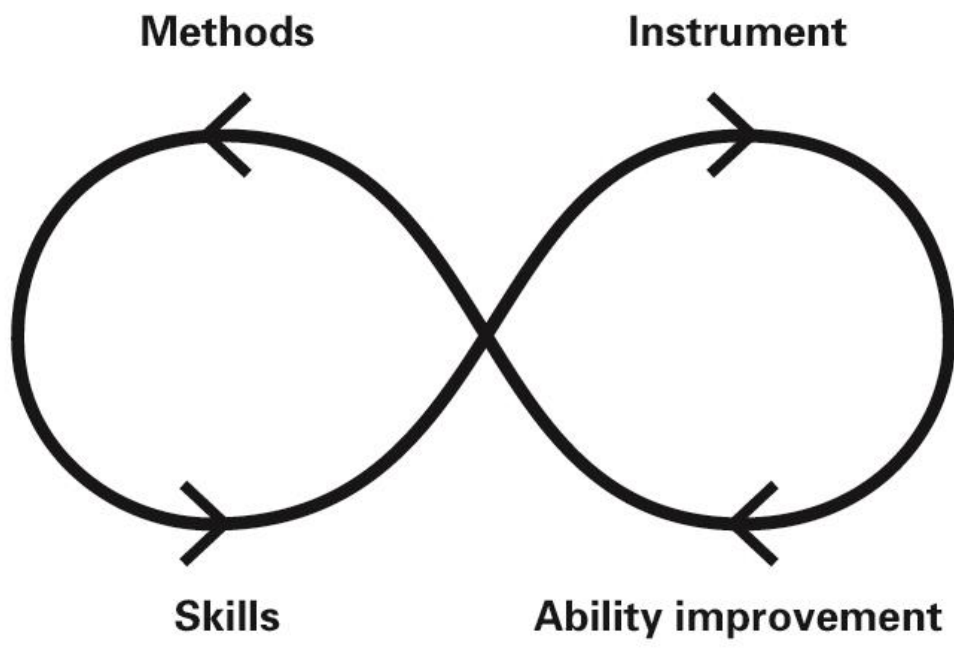

Figure 2 Metaverse in teaching design.

In the study of digital media art as a representative applied subject, in the four years, they mainly learn about learning methods, skills and tools, career and realization ability, and cognition of future development. So, the existence of "metaverse" in any link can be discovered, which is a kind of uncertainty. People are basically not sure what changes it will have in the future, and they are not sure what results it will have, so what they have to do is that in the future, the teachers and students need to adapt to this ever-changing learning environment. That is to say, students' learning is easily affected by technological products, and teachers also need to constantly update and iterate the original knowledge, so as to form the learning motivation of continuous development, change and progress. This motivation is often passive rather than active. It is necessary to consider a problem, that is, how to face the existence of the "metaverse" and transform this passivity into initiative.

The first is in teaching methods or thinking training, it is necessary to intervene in the cultivation of data artificial intelligence and other awareness of technological innovation, just like the use of research tools in research. At present, people are more inclined to use big data for analysis instead of traditional questionnaire survey methods. Here, it is not a denial of the traditional reliability 
advantages of questionnaire surveys, but the usage of big data has more advantages from the perspective of efficiency and data volume. That is, the data service itself can generate great value, so people have to use methods to collect, sort and process data. It is not only a change in thinking, but also a need for development.

The second is in the use of skills training. Traditional hands-on ability is mainly embodied in the use experience accumulated by repeated or indepth social experience, and then the experience is used to judge and design the method, process and result of realization. This approach is because new technologies have not appeared. Just like when AI voice assistance appears in language learning to help, many ways and methods of language learning are broken. For young people, they prefer this way because it means simple, fast and convenient. Therefore, the auxiliary functions that similar technologies bring to solving social life problems often impact traditional learning content and methods. In particular, many aspects of technology application or training have often broken through campus restrictions, but are open to the whole society, which has brought a lot of pressure on campus teaching. Therefore, the role of the teacher becomes a bridge role, converting knowledge into information output and application scenarios.

The third is about the cultivation of career and realization ability. The biggest difference between humans and animals is that humans can use tools, and the biggest difference between modern people and people in the past is that people can use tools to create new tools. The use of this tool will produce several kinds of changes and results. If it is not used innovatively, it will be retrogression. Therefore, no era of artistic design creativity has the convenience and possibility like today. This kind of convenience and may not be available in the past, but also needed in the future. This is probably the "metaverse" as the authors understand it.

In the description of the future career, what people fancy more should be the awareness of innovation, the attitude of innovation and the understanding and judgment of the future based on technical conditions, or the "understanding of fashion and trends" mentioned in the recruitment information. If it is said that specific job requirements must be described in a more specific language, there is no room for imagination. At this time, people want to see "uncertainty" rather than "certainty".

\section{EVERYONE IS CREATING THE METAVERSE}

What does the metaverse uncertainty of digital media art design look like? The combination of technology and art innovation itself does not need to be repeated. It is more appropriate to use science to combine with art, because science is defined as breaking away from the constraints of computers. Biotechnology, photoelectric technology, chemical technology, quantum technology, etc. may affect the specific expression of art or affect the technical standards of design. Therefore, the understanding of the metaverse does not come from the individual but from the entire society. The field that everyone is engaged in may be a part of the metaverse, that is, everyone is a part of the metaverse.

Here, the focus returns to the form of art itself. Whether it is rock paintings or murals on the rocks, or to modern digital painting techniques, it is the pursuit of a unity between art form and recording function. The pursuit of rock painting is documentary and historical records, but the current AI painting is more emphasis on learning the brush touch or artistic sense of human painting. From this point of view, traditional rock paintings have a meaning that transcends time and space, while works produced with modern technology painting restore the authenticity of people to the greatest extent. So, it is necessary to consider finding a relationship between art and technology.

Art is a combination of individual culture and natural things; design is a combination of industry and aesthetics. Because the former has individual subjectivity, technical randomness and result uncertainty, while the latter pays more attention to production rules and standards, process control management and product reliability. People can't imagine what kind of changes the combination of biological gene technology and art will produce. Obviously, it has uncertainty. People can't imagine whether graphene technology can be combined with art to replace traditional gunpowder performance either. Industrial production is unlikely to be handled in this way because it has stricter industrial norms and standards. So what will a mass-produced metaverse look like? While people are looking forward to it, there will be a little regret.

To put it another way, the combination of metaverse and art requires scientific and technological means as a bridge, and the conversion between subjective and objective, art form and 
design rules. The sense of order of industrial products is more in line with the needs of modern life. In other words, people can produce different types of metaverse products through the combination of various science, technology and art. At this time, people will feel that the future is promising, and the "metaverse" turns out to be like this.

Meanwhile, people will also find that the relationship between science, technology and art is actually subjective. Technology plays a method or way to realize conditional functions, science defines the possibility of the development of things, and art is only a spiritual and social need of people.

Therefore, at the practical level, people always have to consider an artistic talent, who will use the program to change what kind of work he will create. Through above analysis, it will be found that it is impossible for one person to complete the superposition of pure art and technology. At this time, the power of interdisciplinary integration is particularly important, so the creation of the metaverse should be the product of social integration.

\section{THE ENLIGHTENMENT OF METAVERSE TO THE DEVELOPMENT OF DESIGN TEACHING}

In what way will the disciplines related to digital media art develop in the future? The development of interdisciplinary integration is emphasized by the construction of new science and new liberal arts. The development of scientific and technological thinking, cognitive methods, and concepts that span time and space, have actually changed the development trend of the humanities and professions[5]. ("Figure 3")

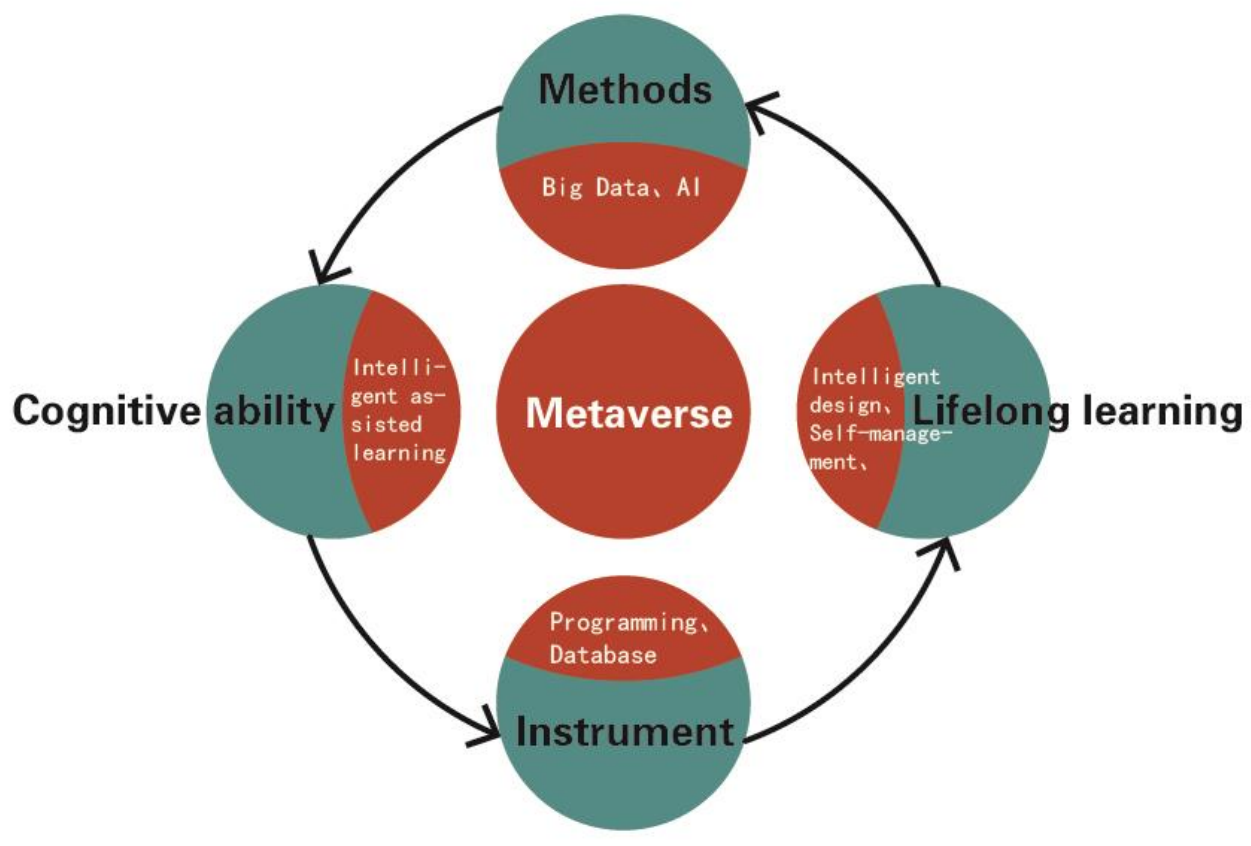

Figure 3 Metaverse in the design of digital media art teaching.

The first is the trend of subject integration and the development of scientific and technological thinking.

The emergence of the metaverse itself represents a trend of technological innovation. Especially when more and more people pay attention to and participate in it, it represents the appeal of technological innovation and requires people not to miss every opportunity for innovation. Therefore, paying attention to the problem itself is the basis of scientific and technological innovation thinking [6].

The second is the improvement of the level of cognition across time and space.

The convenience of scientific and technological progress has directly shortened the distance between people, and the limitations of time and space have been further broken. If the innovation of technological services is to change the spatial 
relationship of the past, individuals with creative thinking need to pay more attention to the ways or possibilities of the temporal and spatial changes of science and technology in the future, and use technological tools to give the possibility of realization.

The third is the intervention of tools and learning methods.

The development and design application of smart tools have performed incisively and vividly in the current design technology environment, and even caused people to change their views on some occupations. For example, artificial intelligenceassisted design systems are preempting traditional visual design methods, and artificial intelligence productivity enhancements are impacting traditional school environments in the Chinese mainland, including campuses. The development process from cultivating basic professional ability to possessing innovative ability is being changed by external technological innovation.

The fourth is the long-term learning and selfimprovement.

It can be seen that the traditional way of competition is mainly between people, but the current competition is between people and machines. This is bound to affect the traditional preference for low-value-added manual labor and repetitive labor, forming a huge social problem. For individuals, the biggest change lies in the improvement of their own sustainable learning ability, actively adapting to the changing technological environment trends, and seeking new growth points, such as positions similar to map data collection.

\section{CONCLUSION}

The metaverse itself is not a technology, but an idea and concept. It needs to integrate different new technologies, a social form and a digital living space where the virtual space and the display world are integrated. The emergence of the metaverse represents the way that humans will change the way of future development through science and technology. It has a great impact on the teaching represented by the digital media art major, and may be changing the design of the teachers' original teaching content. For the instructional design, it is also necessary to actively consider the impact of changes in the technological environment for the realization of the goal of training applied design talents. Therefore, the attention to metaverse is of great significance to the professional development of digital media art.

\section{AUTHORS' CONTRIBUTIONS}

Yong Li wrote the manuscript, and Dan Xiong contributed to revising and editing.

\section{REFERENCES}

[1] Zhang Qian, What Exactly Is "Metaverse"? Why Is It Popular? [N]. China Youth Daily, 2021-11-16(012).

DOI:10.38302/n.cnki.nzgqn. 2021. 004106. (in Chinese)

[2] Yan Yong, Scholars from Many Countries Pay Careful Attention to the Development Trend of "Metaverse" [N]. Chinese Social Sciences Today, 2021-11-05(003). DOI:10.28131/n.cnki.ncshk.2021.004042. (in Chinese)

[3] Liu Geping, Wang Xing, Gao Nan, Hu Hanlin, From Virtual Reality to Metaverse: A New Direction of Online Education [J]. Modern Distance Education Research, 2021, v.33; No.174 (06):12-22. (in Chinese)

[4] Hu Zhe, Wen Jinghua, Why Pay Attention to Metaverse [N]. Economic Information Daily, 2021-11-25(006).

DOI:10.28419/n.cnki.njjck.2021.006442. (in Chinese)

[5] Liu Geping, Wang Xing, Reshaping Online Education by Virtual Reality: Learning Resources, Teaching Organization and System Platform [J]. China Educational Technology, 2020, No. 406(11): 87-96. (in Chinese)

[6] Cao Peijie, Smart Education: The Educational Reform at the Age of Artificial Intelligence [J]. Educational Research, 2018, v.39; No.463(08): 121-128. (in Chinese) 